\title{
Clinical outcomes in Caucasian patients with polypoidal choroidal vasculopathy
}

\author{
Eleftherios I. Agorogiannis $\mathbb{D}^{1} \cdot \operatorname{lan}$ A. Pearce ${ }^{1} \cdot$ Sohraab Yadav $\mathbb{D}^{1} \cdot$ David G. Parry $^{1} \cdot$ Nicholas A. V. Beare $^{1}$
}

Received: 22 January 2018 / Revised: 13 June 2018 / Accepted: 15 June 2018 / Published online: 12 July 2018

(c) The Royal College of Ophthalmologists 2018

\begin{abstract}
Purpose To describe treatment outcomes in a cohort of Caucasian patients with polypoidal choroidal vasculopathy (PCV). Methods Clinical charts from 48 eyes of 45 Caucasian patients with PCV were retrospectively reviewed. All cases were diagnosed with indocyanine green angiography. Best corrected visual acuity (BCVA) and optical coherence tomography (OCT) imaging were analyzed at baseline and final follow-up.

Results Eyes were treated with a combination of verteporfin photodynamic therapy (PDT) and anti-vascular endothelial growth factor (VEGF) $(n=24)$, or PDT monotherapy $(n=9)$, or anti-VEGF monotherapy $(n=8)$, or no treatment $(n=7)$. Aflibercept was the anti-VEGF agent in 30 out of 32 eyes. Sixteen out of 24 eyes in the combination treatment group received initial PDT at diagnosis. All treatments led to stabilization of BCVA at final visit with a trend for better visual acuity in the anti-VEGF monotherapy group. There was a substantial reduction in central retinal thickness associated with resolution of subfoveal fluid and improvement in retinal pigment epithelial detachment in all treatment groups. BCVA and OCT findings remained stable in eyes which received no treatment. The use of PDT was associated with 0.5 fewer intravitreal injections per annum, which was not statistically significant.

Conclusions In the largest series of Caucasian patients with PCV presented to date, anti-VEGF monotherapy, PDT, or their combination preserved visual acuity and improved subfoveal exudative changes. Combination treatment was not superior to anti-VEGF monotherapy.
\end{abstract}

\section{Introduction}

Polypoidal choroidal vasculopathy (PCV) is characterized by a branching network of anomalous inner choroidal vessels with terminal aneurysmal dilatations, which can be associated with serous and/or hemorrhagic retinal pigment epithelium (RPE) or neurosensory retinal detachments $[1,2]$. PCV is commonly regarded as a variant of neovascular age-related macular degeneration (AMD), nevertheless the identification of discrete demographic, clinical, and angiographic features supports its classification as a

Electronic supplementary material The online version of this article (https://doi.org/10.1038/s41433-018-0168-2) contains supplementary material, which is available to authorized users.

Eleftherios I. Agorogiannis

eleftherios.agorogiannis@gmail.com

1 St Paul's Eye Unit, Royal Liverpool University Hospital, Liverpool, United Kingdom distinct clinical entity $[2,3]$. Patients with PCV tend to present at a younger age with mainly unilateral disease, and extra-macular involvement (predominantly juxtapapillary) is frequent $[2,3]$.

The utilization of indocyanine green angiography (ICGA) as the gold standard for the diagnosis of PCV has augmented the recognition of this choroidal disorder [4]. Originally described as a disease typically affecting black females [1], PCV has now been identified in multiple ethnic groups and male patients as well $[2,5]$. Prevalence is particularly high within East Asian populations with reported rates of 22.3-61.6\% among patients with presumed neovascular AMD [5]. Interestingly, a male preponderance has been recognized among Asian patients with PCV [5], but is not observed in Caucasian populations [2], where the overall prevalence of PCV appears to be lower. Prevalence ranges between 4 and $16.8 \%$ among individuals with suspected wet AMD in different Caucasian cohorts [3, 6-11], and was estimated at $8.7 \%$ in a recent meta-analysis [12]. Of note, in a group of Caucasians who responded poorly to intravitreal ranibizumab, frequency of PCV was $21.5 \%$ [11]. 
Current available therapeutic options for PCV include verteporfin photodynamic therapy (PDT) and antivascular endothelial growth factor (anti-VEGF) injections [2, 5, 13-17]. The EVEREST study was the first randomized clinical trial to compare the effects of PDT, anti-VEGF, and their combination in patients with PCV [18]. PDT, with or without ranibizumab, was superior to ranibizumab monotherapy in inducing regression of polyps at 6 months [18]. However, despite the persistence of angiographic findings, patients treated only with ranibizumab still experienced improved visual acuity, comparable to the other two groups [18]. Published recommendations on the treatment of PCV with macular involvement suggested the use of ICGA-guided PDT with or without ranibizumab [19], and a subsequent randomized trial highlighted the superiority of initial (as compared with deferred) PDT in minimizing the need for further intravitreal ranibizumab [20]. With the introduction of aflibercept as an anti-VEGF agent in the treatment of neovascular AMD, its effect on PCV has been studied. Aflibercept monotherapy was associated with favorable visual and anatomical outcomes when used as a first line agent in treatment-naïve patients [21-23] or as salvage treatment in PCV resistant to ranibizumab $[24,25]$.

Despite this information from clinical studies on management of PCV with PDT and/or different antiVEGF agents, an important caveat remains as the bulk of relevant evidence (including the EVEREST trial) was derived from studies performed in Asian patients. In fact, different ethnic groups may present with disparate clinical features of PCV, as exemplified by the higher frequency of unilateral and macular disease in Asian patients $[2,5,26]$. Although there is currently no evidence to suggest significant underlying genetic disparities, the vast majority of association studies reported so far have been performed in Asians [27]. Therefore, the possibility of differential treatment responses between Asian and Caucasian populations with PCV cannot be excluded. There is a relative paucity of clinical data on treating PCV in Caucasian patients, mainly owing to the lower disease prevalence in these populations. In a retrospective study of Caucasian Greek treatment-naive patients, PDT was associated with better visual outcomes after 1 year [28], and two additional series of white American [10] and Swiss [11] patients supported the use of PDT in PCV nonresponsive to intravitreal anti-VEGF treatment. As ranibizumab was the predominant anti-VEGF agent used in these reports, limited evidence exists regarding the use of aflibercept in Caucasians with PCV.

Additional information on treatment results in Caucasian patients is undoubtedly needed. We recently reported a PCV prevalence of $9.1 \%$ among Caucasians with presumed neovascular AMD, but $22.1 \%$ among those classified as occult neovascularization [7]. The aim of the current study was to describe the clinical outcomes in our cohort following treatment with PDT, anti-VEGF or both. We present real world data that demonstrate effectiveness of different treatment modalities in Caucasian patients with PCV, and add to the clinical evidence about the use of aflibercept in this population.

\section{Patients and methods}

\section{Study population and inclusion criteria}

The clinical notes of 46 non-consecutive Caucasian patients (group overlapping with the cohort we previously reported [7]) diagnosed with PCV between December 2013 and March 2017 were retrospectively reviewed. The study was approved by the audit department of the Royal Liverpool University Hospital and conformed to the standards described in the declaration of Helsinki.

\section{Visual acuity measurement and imaging}

All patients underwent fundus ICGA. Diagnosis was established by a panel of at least two retina experts following consensus on the presence of specific angiographic features as described by the EVEREST study group [29]. Best corrected visual acuity (BCVA) was measured in early treatment diabetic retinopathy study (ETDRS) letters with refraction at baseline and each clinic visit. Spectral domain (SD) optical coherence tomography (OCT) imaging of the macula was performed at baseline and each clinic visit using Spectralis SD-OCT (Heidelberg Engineering). The presence of pigment epithelial detachment (PED), subretinal fluid (SRF), and intraretinal fluid (IRF) was noted. Central retinal thickness (CRT) was derived from the central 1-mm ETDRS subfield using Spectralis segmentation. Macular imaging on two patients was performed with the Topcon 3D OCT-2000 at final visit; only information on the presence of PED, SRF and IRF was used, and the relevant CRT values (as well as the paired initial values obtained using Spectralis) were excluded from analysis.

\section{Treatment}

Once a diagnosis was made based on multimodal imaging, the same retina specialists suggested an initial management plan, nevertheless treatment modality ultimately depended on the managing physician's discretion and the patient's preference following informed consent. Patients were treated with verteporfin (Visudyne, Novartis) PDT, or intravitreal anti-VEGF injections, or a combination of the two treatments, or received no treatment if appropriate. 
Anti-VEGF agents included either ranibizumab (Lucentis, Novartis) or aflibercept (Eylea, Bayer). Patients with PED, IRF, or SRF affecting the central macula were recommended combination treatment or anti-VEGF monotherapy. Patients without central macular involvement, who nevertheless had lesions that threatened the central macula, were recommended PDT. Other patients were recommended observation. The number of anti-VEGF injections or PDT sessions required was recorded. One patient treated with a combination of PDT and anti-VEGF injections was not included in these calculations due to short follow-up of only 8.1 weeks. Two patients managed with PDT had history of prior anti-VEGF treatment (first patient initially diagnosed as minimally classic choroidal neovascularization; second patient diagnosed with recurrence of PCV which had been treated with anti-VEGF $>4$ years before). Both patients were included in the PDT monotherapy group as they received no further intravitreal injections.

\section{Statistical analysis}

Where indicated, statistical analysis was performed using GraphPad Prism for Mac (version 5.0a, Graphpad Software, San Diego, CA, USA) with Student's $t$ test, Wilcoxon signed rank test, or Mann-Whitney test as appropriate, depending on the result of the D'AgostinoPearson normality test. $P$ values $<0.05$ were classified as significant.

\section{Results}

\section{Baseline characteristics}

Clinical charts from 49 eyes of 46 Caucasian patients were retrospectively reviewed. The initial characteristics of the patient population are presented in Table 1. Median age was 76 years and there was a female preponderance (63\%). Forty-four eyes received a new diagnosis of PCV, whereas five eyes presented with either recurrent PCV or neovascular AMD refractory to intravitreal anti-VEGF. Mean initial visual acuity was 69.4 letters. Thirty-six eyes $(73.5 \%)$ were phakic at presentation; three eyes underwent cataract surgery before final follow-up. One patient died soon after initial assessment and was excluded from further analysis.

\section{Patient groups and treatments}

Forty-eight eyes of 45 Caucasian patients were studied following initial assessment. Treatment included a combination of PDT and anti-VEGF (24 eyes, 50\%), or PDT alone ( 9 eyes, $18.8 \%$ ), or anti-VEGF monotherapy (8 eyes,
Table 1 Baseline characteristics of total patient population

\begin{tabular}{ll}
\hline Total number of patients & 46 \\
Median age (range) & 76 years $(51-89)$ \\
Sex & \\
Male $(\%)$ & $17(37.0 \%)$ \\
Female (\%) & $29(63.0 \%)$ \\
Patients with bilateral disease $(\%)$ & $3(6.5 \%)$ \\
Total number of eyes & 49 \\
Right/left eye & $25 / 24$ \\
Mean initial BCVA $( \pm \mathrm{SD})$ & $69.4 \pm 10.1$ letters \\
Lens status & \\
Phakic eyes $(\%)$ & $36(73.5 \%)^{\mathrm{a}}$ \\
Pseudophakic eyes $(\%)$ & $13(26.5 \%)$ \\
\hline
\end{tabular}

$B C V A$ best corrected visual acuity; $S D$ standard deviation

${ }^{\text {a }}$ Three eyes had cataract surgery following initial assessment and before final follow-up

16.7\%). Seven eyes (14.6\%) received no treatment. The clinical characteristics of each group are presented in Table 2. As expected, mean baseline BCVA was highest among eyes that received no treatment, and eyes treated with PDT alone also presented with good initial visual acuity. Eyes in these two groups were more likely to have juxtapapillary lesions. On the contrary, macular lesions were more common among eyes that received combination treatment or anti-VEGF monotherapy, and this mirrored the lower mean baseline BCVA in these two treatment groups.

Aflibercept was used in the vast majority of patients treated with anti-VEGF except for three in the combination treatment group (two treated with ranibizumab only, and one treated initially with ranibizumab which was eventually switched to aflibercept). All eyes treated with anti-VEGF received a total of three intravitreal injections every 4 weeks initially, with the exception of one eye, which received only one aflibercept injection in the combination treatment group. Following loading, the decision was made to continue with either fixed interval regimen or variable dosing (as needed or treat-and-extend), or observation.

Timing of PDT and anti-VEGF injections in the combination treatment group varied according to clinical need and decision-making. Fourteen eyes received concurrent PDT and anti-VEGF injections. Seven eyes were initially treated with anti-VEGF, and PDT was introduced later due to insufficient response to intravitreal injections. Finally three eyes were treated with PDT initially, before antiVEGF therapy was given.

No serious complications were observed in eyes that received PDT only or no treatment at all. Two eyes treated with anti-VEGF (one in each of the combination and monotherapy groups) suffered RPE tears. 
Table 2 Clinical characteristics of the eyes included in the analysis

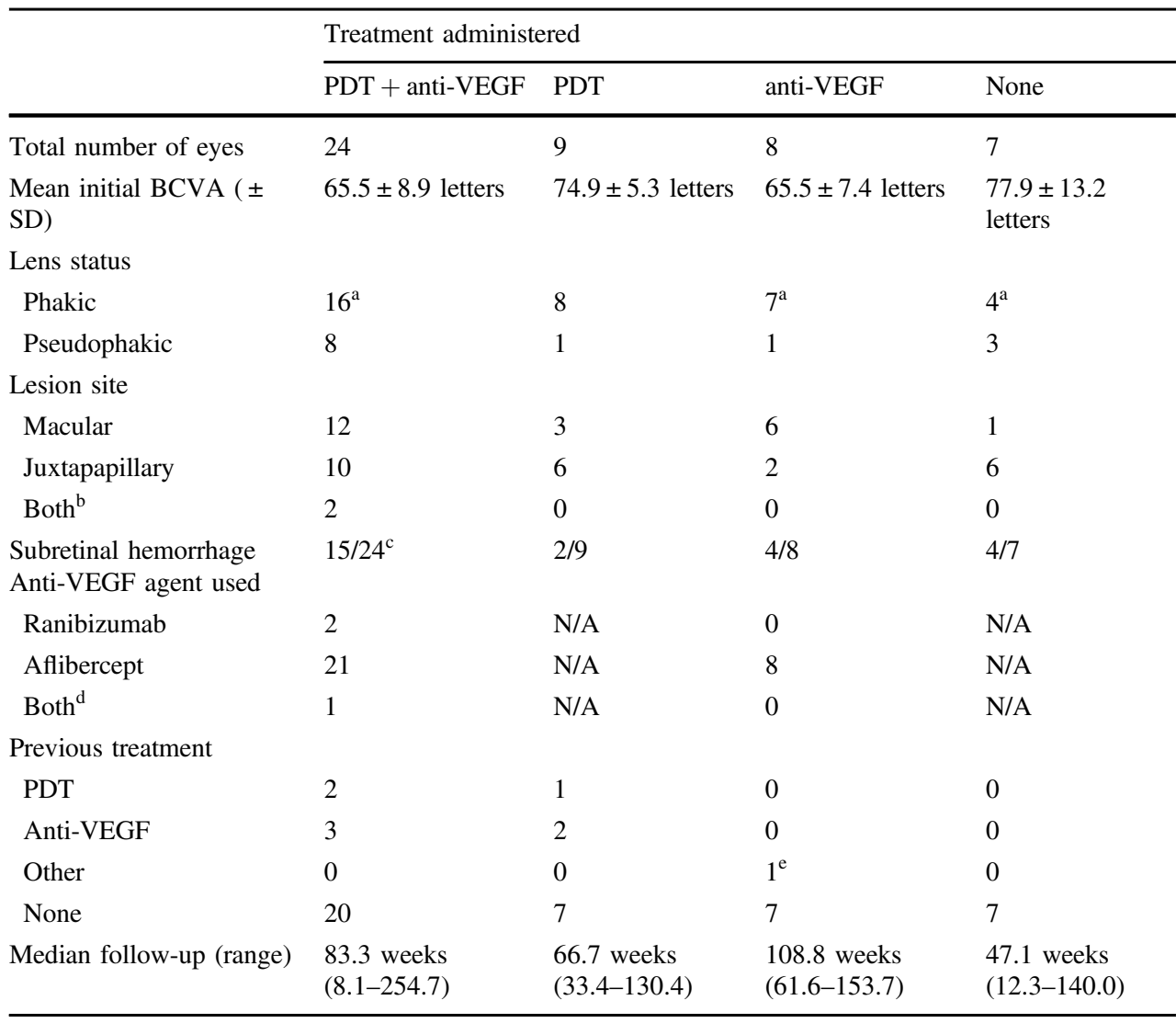

$B C V A$ best corrected visual acuity, $S D$ standard deviation, $N / A$ not applicable

${ }^{a}$ One eye in each of these groups had cataract surgery following initial assessment and before final follow-up ${ }^{b}$ Polyp identified at the junction between macula and juxtapapillary area

${ }^{c}$ One patient developed submacular hemorrhage after initial presentation

${ }^{\mathrm{d}}$ Treatment initiated with ranibizumab, subsequently switched to aflibercept

e Large submacular hemorrhage treated as an emergency with intravitreal tissue plasminogen activator and bevacizumab

\section{Effect of treatment on BCVA and exudative pathology, and final outcomes}

Eyes in no treatment, PDT alone and combination therapy groups experienced no significant change of BCVA on average at final follow-up. The anti-VEGF monotherapy group had a statistically significant increase in BCVA of 5.3 letters at final clinic visit. There was a significant reduction in CRT in all treatment groups, and these changes were accompanied by reduction in the levels of SRF and/or IRF, resolution of subfoveal exudative changes and improvement of PED. Eyes that received no treatment showed no significant change in CRT and macular pathology.

Total median follow-up was 81.9 weeks. At final visit, 10 out of $24(41.7 \%)$ eyes in the combination treatment group were still receiving anti-VEGF injection, compared with 6 out of $8(75 \%)$ eyes in the aflibercept monotherapy group. All patients treated with PDT only had either been discharged or entered a period of monitoring.

Table 3 summarizes treatment outcomes. A series of clinical examples from our patient series are presented as supplementary material (supplementary figures 1-6).

\section{Frequency of PDT and intravitreal anti-VEGF treatment}

The average number of intravitreal injections during the first year in the combination treatment and anti-VEGF monotherapy groups, was 5.5 and 6.0, respectively $(p=0.5990$; Table 4). Similarly, the average number of injections per year did not differ between these two groups (4.6 and 5.1, respectively, $p=0.6243$ ). We additionally calculated the number of intravitreal injections in 16 patients of the combination treatment group that received PDT at initial 
Table 3 Changes in BCVA and exudative pathology and final outcomes

\begin{tabular}{|c|c|c|c|c|}
\hline & \multicolumn{4}{|c|}{ Treatment administered } \\
\hline & $\begin{array}{l}\text { PDT + anti-VEGF } \\
(n=24)\end{array}$ & $\operatorname{PDT}(n=9)$ & $\begin{array}{l}\text { Anti-VEGF } \\
(n=8)\end{array}$ & None $(n=7)$ \\
\hline \multicolumn{5}{|l|}{ Mean BCVA $( \pm$ SD $)$} \\
\hline Baseline & $65.5 \pm 8.9$ letters & $\begin{array}{l}74.9 \pm 5.3 \\
\text { letters }\end{array}$ & $65.5 \pm 7.4$ letters & $77.9 \pm 13.2$ letters \\
\hline Final visit & $66.4 \pm 14.8$ letters & $\begin{array}{l}75.2 \pm 5.8 \\
\text { letters }\end{array}$ & $70.8 \pm 7.9$ letters & $76.9 \pm 9.4$ letters \\
\hline$p^{\mathrm{a}}$ & 0.2317 & 0.6733 & 0.0343 & 0.4004 \\
\hline $\begin{array}{l}\text { Mean BCVA change } \\
( \pm \text { S D })\end{array}$ & $0.8 \pm 11.1$ letters & $0.3 \pm 3.6$ letters & $5.3 \pm 4.9$ letters & $-1.0 \pm 13.8$ letters \\
\hline \multicolumn{5}{|l|}{ Mean CRT $( \pm$ SD $)$} \\
\hline Baseline & $422.0 \pm 161.6 \mu \mathrm{m}^{\mathrm{b}}$ & $\begin{array}{l}335.0 \pm 160.2 \\
\mu \mathrm{m}\end{array}$ & $473.6 \pm 194.8 \mu \mathrm{m}$ & $300.3 \pm 36.0 \mu \mathrm{m}$ \\
\hline Final visit & $244.9 \pm 42.1 \mu \mathrm{m}^{\mathrm{b}}$ & $\begin{array}{l}284.2 \pm 132.5 \\
\mu \mathrm{m}\end{array}$ & $266.3 \pm 60.9 \mu \mathrm{m}$ & $298.4 \pm 54.8 \mu \mathrm{m}$ \\
\hline$p^{\mathrm{a}}$ & $<0.0001$ & 0.0151 & 0.0156 & 0.5992 \\
\hline \multicolumn{5}{|l|}{ SRF and/or IRF ( $n$ eyes) } \\
\hline Present at baseline & 24 & 8 & 8 & 1 \\
\hline Present at final visit & 6 & 4 & 5 & 1 \\
\hline \multicolumn{5}{|l|}{ Subfoveal fluid ( $n$ eyes) } \\
\hline Present at baseline & 18 & 4 & 7 & 0 \\
\hline Present at final visit & 2 & 1 & 2 & 0 \\
\hline \multicolumn{5}{|l|}{ PED ( $n$ eyes) } \\
\hline Present at baseline & 23 & 8 & 8 & 2 \\
\hline Improved at final visit & 20 & 6 & 6 & 1 \\
\hline \multicolumn{5}{|l|}{ Status at final visit } \\
\hline Discharged & 2 & 2 & 0 & 3 \\
\hline Monitoring & 12 & 7 & 2 & 4 \\
\hline Fixed interval dosing & 4 & N/A & 0 & N/A \\
\hline Treat-and-extend & 6 & N/A & 6 & N/A \\
\hline
\end{tabular}

$B C V A$ best corrected visual acuity, $S D$ standard deviation, $C R T$ central retinal thickness, $S R F$ subretinal fluid, $I R F$ intraretinal fluid, PED pigment epithelial detachment, N/A not applicable

${ }^{\mathrm{a}}$ Comparison of baseline and final BCVA and CRT values in the same treatment group (two-tailed Wilcoxon signed rank test)

${ }^{\mathrm{b}}$ Mean CRT values calculated from $n=22$ eyes clinic visit (simultaneously with or before the start of antiVEGF therapy). These patients received on average 4.7 injections during the first year and 4.3 injections per year. Comparison of these numbers with the anti-VEGF monotherapy group did not demonstrate a statistically significant difference. The use of anti-VEGF agents was associated with fewer total PDT treatments in the combination group (1.2) versus PDT alone (1.7) (Table 4).

\section{Clinical outcomes in patients with juxtapapillary PCV}

We further analyzed clinical characteristics and outcomes in 24 eyes with juxtapapillary polypoidal lesions, which generally present with a higher frequency among Caucasian populations [2]. Baseline characteristics and final outcomes are summarized in Table 5. Decision for treatment was made in 18 out of 24 eyes $(75 \%)$ with exudative changes, whereas observation was recommended in six eyes $(25 \%)$ with no SRF or IRF at baseline. Most treated eyes received PDT, either alone $(6 / 18,33.3 \%)$ or in combination with anti-VEGF $(10 / 18,55.6 \%)$, whereas two eyes $(11.1 \%)$ were treated with aflibercept monotherapy. Treatment induced an overall improvement of exudative changes and a corresponding decrease in CRT, and was associated with an increase in average BCVA of 2.6 letters, which was not statistically significant. Meanwhile, untreated eyes with juxtapapillary PCV remained stable with no significant change in BCVA 
Table 4 Numbers of intravitreal anti-VEGF injections and PDT sessions

\begin{tabular}{|c|c|c|c|c|}
\hline & \multicolumn{3}{|c|}{ Treatment group } & \multirow[t]{2}{*}{$p^{\mathrm{a}}$} \\
\hline & $\begin{array}{l}\text { PDT + anti- } \\
\text { VEGF }\end{array}$ & PDT & $\begin{array}{l}\text { anti- } \\
\text { VEGF }\end{array}$ & \\
\hline $\begin{array}{l}\text { Number of } \\
\text { injections }\end{array}$ & (all patients) & & & \\
\hline Total & $8.5 \pm 5.6$ & N/A & $10.5 \pm 4.8$ & 0.382 \\
\hline During first year & $5.5 \pm 2.4$ & N/A & $6.0 \pm 2.4$ & 0.5990 \\
\hline Annual rate & $4.6 \pm 2.1$ & N/A & $5.1 \pm 2.3$ & 0.624 \\
\hline $\begin{array}{l}\text { Number of } \\
\text { injections }\end{array}$ & $\begin{array}{l}\text { (PDT at } \\
\text { baseline) }\end{array}$ & & & \\
\hline Total & $7.8 \pm 5.8$ & N/A & $10.5 \pm 4.8$ & 0.2611 \\
\hline During first year & $4.7 \pm 2.0$ & N/A & $6.0 \pm 2.4$ & 0.1680 \\
\hline Annual rate & $4.3 \pm 2.0$ & N/A & $5.1 \pm 2.3$ & 0.419 \\
\hline $\begin{array}{l}\text { Number of PDT } \\
\text { sessions }\end{array}$ & (all patients) & & & \\
\hline Total & $1.2 \pm 0.5$ & $1.7 \pm 0.7$ & N/A & 0.0402 \\
\hline Annual rate & $0.8 \pm 0.5$ & $1.3 \pm 0.7$ & N/A & 0.0455 \\
\hline
\end{tabular}

Numbers of treatments are all presented as mean \pm standard deviation N/A not applicable

${ }^{a}$ Comparison of the number of treatments between the two groups in each row as applicable. Two-tailed unpaired Student's $t$ test was used for the number of injections and two-tailed Mann-Whitney test was used for the number of PDT sessions

and CRT, and required no therapy. At last clinic visit, only 1 out of 18 treated eyes $(5.6 \%)$ was still receiving anti-VEGF injections using a treat-and-extend regimen.

\section{Discussion}

We retrospectively studied the clinical outcomes following treatment or observation in a large series of Caucasian patients with PCV. We demonstrated the effectiveness of PDT, anti-VEGF, and their combination in maintaining visual acuity and improving exudative changes in the majority of eyes. We found no evidence that combination therapy with PDT and anti-VEGF was superior to antiVEGF alone; on the contrary, anti-VEGF monotherapy showed better visual outcomes compared to combination treatment (albeit without randomization). However, where PDT could be used alone it was a suitable therapy which avoided anti-VEGF injections with a mean total of 1.7 PDT treatments. Baseline patient characteristics were comparable to those described in previous cohorts of Caucasians with PCV with mainly unilateral involvement and a high frequency of juxtapapillary lesions $[6,8-10,28]$.

Mean BCVA at presentation was higher among eyes that received PDT or no treatment, as these patients had absent
Table 5 Subgroup analysis of patients with juxtapapillary PCV

\begin{tabular}{|c|c|c|}
\hline & Treated & Untreated \\
\hline Total number of eyes & 18 & 6 \\
\hline $\begin{array}{l}\text { Median follow-up } \\
\text { (range) }\end{array}$ & $\begin{array}{l}68.4 \text { weeks }(8.1 \pm \\
116.0)\end{array}$ & $\begin{array}{l}57.6 \text { weeks } \\
(12.3-140.0)\end{array}$ \\
\hline \multicolumn{3}{|l|}{ Treatment } \\
\hline $\begin{array}{l}\text { Anti-VEGF and } \\
\text { PDT }\end{array}$ & 10 & N/A \\
\hline PDT alone & 6 & N/A \\
\hline Anti-VEGF alone & 2 & N/A \\
\hline \multicolumn{3}{|l|}{ Mean BCVA $( \pm$ SD) } \\
\hline Baseline & $70.1 \pm 8.7$ letters & $78.3 \pm 14.4$ letters \\
\hline Final visit & $72.7 \pm 8.4$ letters & $77.7 \pm 10.1$ letters \\
\hline$p^{\mathrm{a}}$ & 0.0740 & 0.5879 \\
\hline $\begin{array}{l}\text { Mean BCVA change } \\
( \pm \text { SD })\end{array}$ & $2.6 \pm 5.5$ letters & $-0.7 \pm 15.1$ letters \\
\hline \multicolumn{3}{|l|}{ Mean CRT $( \pm$ SD) } \\
\hline Baseline & $351.1 \pm 110.8 \mu \mathrm{m}^{\mathrm{b}}$ & $289.0 \pm 22.0 \mu \mathrm{m}$ \\
\hline Final visit & $242.3 \pm 26.7 \mu \mathrm{m}^{\mathrm{b}}$ & $301.5 \pm 59.4 \mu \mathrm{m}$ \\
\hline$p^{\mathrm{a}}$ & 0.0004 & 0.8923 \\
\hline \multicolumn{3}{|c|}{ SRF and/or IRF ( $n$ eyes) } \\
\hline Present at baseline & 18 & 0 \\
\hline Present at final visit & 6 & 1 \\
\hline \multicolumn{3}{|c|}{ Subfoveal fluid ( $n$ eyes) } \\
\hline Present at baseline & 11 & 0 \\
\hline Present at final visit & 0 & 0 \\
\hline \multicolumn{3}{|l|}{ PED ( $n$ eyes) } \\
\hline Present at baseline & 16 & 1 \\
\hline $\begin{array}{l}\text { Improved at final } \\
\text { visit }\end{array}$ & 13 & 0 \\
\hline \multicolumn{3}{|l|}{ Status at final visit } \\
\hline Discharged & 2 & 2 \\
\hline Monitoring & 15 & 4 \\
\hline Treat-and-extend & 1 & N/A \\
\hline
\end{tabular}

N/A not applicable, BCVA best corrected visual acuity, $S D$ standard deviation, $C R T$ central retinal thickness, $S R F$ subretinal fluid, IRF intraretinal fluid, $P E D$ pigment epithelial detachment

${ }^{\mathrm{a}}$ Comparison of baseline and final BCVA and CRT values in the same treatment group (two-tailed Wilcoxon signed rank test)

${ }^{\mathrm{b}}$ Mean CRT values calculated from $n=17$ eyes

central SRF and exudative changes and they were more likely to have juxtapapillary lesions. On the other hand, eyes with central macular involvement with subfoveal exudative changes were generally treated with anti-VEGF, with or without PDT. In essence, it would seem a reasonable approach to target severe fluid leakage with an anti-VEGF agent, but it is likely that the lower starting visual acuity in these subgroups influenced the physician and patient's decision toward engaging into more aggressive management with intravitreal injections. Patients with macular or perimacular polypoidal 
lesions but without central macular involvement can be treated with PDT alone, certainly in the first instance.

Aflibercept was the preferred anti-VEGF agent in most cases. In fact, a growing body of evidence supports the use of aflibercept monotherapy in Asians with PCV, especially in eyes with recalcitrant disease that no longer responds to ranibizumab [24, 25]. In a retrospective study of Caucasian patients in Denmark, the initial change in visual acuity and CRT did not persist long-term, and 7 out of 29 eyes required salvage PDT [30]. Contrary to this finding, we observed long-lasting anatomical improvements with aflibercept treatment and a higher requirement for PDT compared with the Danish study. Different clinical features in the Danish cohort, including a high frequency of extramacular lesions (62\%) [30], may account for this discrepancy. BCVA improvement following aflibercept monotherapy in our study was significantly greater than the other treatment groups, however, owing to the low number of eyes in this subgroup, we avoid overstating the clinical importance of this finding.

With the exception of one case, all eyes treated with antiVEGF received a loading course of three intravitreal injections, followed by either monitoring or further treatment with a fixed or variable dosing regimen. Fixed interval therapy followed the treatment schedule introduced by the VIEW 1 and 2 studies for the use of aflibercept in neovascular AMD [31], with an intention for a total of seven injections during the first year after diagnosis. Fixed interval aflibercept monotherapy has been found to confer superior visual outcomes compared with as needed treatment in Asians with PCV [22, 23], nevertheless issues such as treatment burden and patient compliance are important considerations in real life clinical settings. A treat-andextend therapy pattern may offer a useful compromise, but at some stage monitoring without treatment is initiated followed by as needed treatment.

Thirty-three eyes in our study were treated with verteporfin PDT. Nine eyes received no additional treatment and experienced on average stable visual acuity and significant reduction in CRT at final follow-up. A combination of PDT and intravitreal anti-VEGF was administered to 24 eyes. Significant macular involvement with subfoveal fluid was a critical factor in deciding the use of anti-VEGF treatment. Timing of PDT in the combination treatment group varied, but most patients were treated at the time of their first clinic visit. Both early and delayed PDT have been reported to afford comparable clinical outcomes [20]. Nevertheless, initial treatment in one study was found to decrease the requirement for prolonged intravitreal treatment [20], perhaps by addressing the underlying cause of fluid leakage at early stages. Contrary to previous reports $[11,18]$, we failed to observe a substantial reduction in the need for anti-VEGF in patients who received PDT.
Visual and anatomical outcomes produced by antiVEGF monotherapy were non-inferior to combination treatment in our study, therefore, a reasonable question would pertain to the overall role of PDT in management of PCV. The EVEREST study certainly suggested a beneficial effect of PDT at 6 months [18], however two additional randomized clinical trials (EVEREST II and PLANET) compared anti-VEGF monotherapy with combination treatment. In EVEREST II, patients were randomized into receiving either ranibizumab monotherapy or ranibizumab with PDT; combination treatment was associated with a statistically significant visual acuity gain at 12 months [32]. In the PLANET trial, patients received an initial loading dose of three aflibercept injections, which led to significant anatomical improvement in the majority of patients [33]. Individuals requiring rescue therapy continued receiving aflibercept every 4 weeks and were further randomized into receiving sham or active PDT [33]. At 52 weeks, PDT did not offer a superior functional or anatomical advantage compared with aflibercept monotherapy [33]. As the timing of PDT was different in each setting, the interpretation of the results of these two trials is not straightforward. Accumulating evidence supports the use of aflibercept monotherapy in both Asian [21-23] and Caucasian patients [30] with PCV, however, we believe that PDT still holds a significant place, mainly as monotherapy in selected patients without central macular involvement in whom anti-VEGF injections can then be avoided. PDT was administered in $88.9 \%$ of eyes with juxtapapillary PCV in our study, however the majority of these additionally received antiVEGF injections owing to exudative pathology involving or encroaching on the fovea. Although previous randomized trials studied only individuals with macular PCV [18, 32, 33], aggressive treatment should be considered for active juxtapapillary lesions with central maculathreatening exudative changes [5], and in such settings PDT can be safely delivered with no attendant risk of later foveal atrophy.

As a retrospective analysis of a cohort, our study has inherent weaknesses that we recognize. Most importantly, it was non-randomized and treatment selection was based on clinical features. Nevertheless, there is important information to be drawn.

In summary, we presented real life clinical data on the largest series of Caucasian patients with PCV reported so far. We found no evidence that combination PDT/aflibercept therapy was superior to aflibercept alone, and the latter had better visual outcomes. PDT alone was a suitable treatment where the central macula was not involved. Randomized studies in different settings are needed for definitive data on optimal treatment regimens for PCV taking into account the degree of macular involvement. 


\section{Summary}

\section{What was known before}

- Clinical information on PCV treatment is based mainly on Asian patients in whom PDT, with or without ranibizumab, is superior to ranibizumab monotherapy. Aflibercept monotherapy can be effective in treating PCV in Asians.

- Evidence on treating PCV in Caucasians remains generally scarce, partly due to lower disease prevalence in this population.

- Additional studies on Caucasian patients are required, particularly about the effectiveness of aflibercept with or without PDT.

\section{What this study adds}

- The current study describes clinical outcomes in the largest series of Caucasian patients with PCV presented to date.

- Aflibercept monotherapy, PDT, or their combination can preserve visual acuity and improve subfoveal exudative changes in Caucasians with PCV. Combination treatment is not superior to aflibercept monotherapy.

- PDT can be effective as monotherapy in selected patients without central macular involvement.

Acknowledgements We are indebted to Dr Gabriela Czanner for her advice on statistical analysis.

\section{Compliance with ethical standards}

Conflict of interest Ian A. Pearce has received consultancy fees from Bayer and Novartis and travel grants from Bayer. Nicholas A.V. Beare is a member of the NICE AMD Clinical Guidelines Development Committee, has participated in advisory boards for Santen Pharmaceuticals and AbbVie on uveitis, and has received a travel grant from Bayer.

\section{References}

1. Yannuzzi LA, Sorenson J, Spaide RF, Lipson B. Idiopathic polypoidal choroidal vasculopathy (IPCV). Retina. 1990;10:1-8.

2. Imamura $Y$, Engelbert M, Iida T, Freund KB, Yannuzzi LA. Polypoidal choroidal vasculopathy: a review. Surv Ophthalmol. 2010;55:501-15.

3. Yannuzzi LA, Wong DW, Sforzolini BS, Goldbaum M, Tang KC, Spaide RF, et al. Polypoidal choroidal vasculopathy and neovascularized age-related macular degeneration. Arch Ophthalmol. 1999;117:1503-10.

4. Spaide RF, Yannuzzi LA, Slakter JS, Sorenson J, Orlach DA. Indocyanine green videoangiography of idiopathic polypoidal choroidal vasculopathy. Retina. 1995;15:100-10.
5. Wong CW, Yanagi Y, Lee WK, Ogura Y, Yeo I, Wong TY, et al. Age-related macular degeneration and polypoidal choroidal vasculopathy in Asians. Prog Retin Eye Res. 2016;53:107-39.

6. Scassellati-Sforzolini B, Mariotti C, Bryan R, Yannuzzi LA, Giuliani M, Giovannini A. Polypoidal choroidal vasculopathy in Italy. Retina. 2001;21:121-5.

7. Yadav S, Parry DG, Beare NA, Pearce IA. Polypoidal choroidal vasculopathy: a common type of neovascular age-related macular degeneration in Caucasians. Br J Ophthalmol. 2017;101:1377-80.

8. Ladas ID, Rouvas AA, Moschos MM, Synodinos EE, Karagiannis DA, Koutsandrea CN. Polypoidal choroidal vasculopathy and exudative age-related macular degeneration in Greek population. Eye. 2004;18:455-9.

9. Lafaut BA, Leys AM, Snyers B, Rasquin F, De Laey JJ. Polypoidal choroidal vasculopathy in Caucasians. Graefes Arch Clin Exp Ophthalmol. 2000;238:752-9.

10. Davis SJ, Lauer AK, Flaxel CJ. Polypoidal choroidal vasculopathy in white patients. Retina. 2014;34:2185-91.

11. Hatz K, Prünte C. Polypoidal choroidal vasculopathy in Caucasian patients with presumed neovascular age-related macular degeneration and poor ranibizumab response. $\mathrm{Br} \mathrm{J}$ Ophthalmol. 2014;98:188-94.

12. Lorentzen TD, Subhi Y, Sørensen TL. Prevalence of polypoidal choroidal vasculopathy in white patients with exudative agerelated macular degeneration. Systematic review and metaanalysis. Retina. 2017; https://doi.org/10.1097/IAE. 0000000000001872

13. Nowak-Sliwinska P, van den Bergh H, Sickenberg M, Koh AH. Photodynamic therapy for polypoidal choroidal vasculopathy. Prog Retin Eye Res. 2013;37:182-99.

14. Wong CW, Cheung CM, Mathur R, Li X, Chan CM, Yeo I, et al. Three-year results of polypoidal choroidal vasculopathy treated with photodynamic therapy: retrospective study and systematic review. Retina. 2015;35:1577-93.

15. Spaide RF, Donsoff I, Lam DL, Yannuzzi LA, Jampol LM, Slakter J, et al. Treatment of polypoidal choroidal vasculopathy with photodynamic therapy. Retina. 2002;22:529-35.

16. Cho HJ, Kim JW, Lee DW, Cho SW, Kim CG. Intravitreal bevacizumab and ranibizumab injections for patients with polypoidal choroidal vasculopathy. Eye. 2012;26:426-33.

17. Kokame GT, Yeung L, Lai JC. Continuous anti-VEGF treatment with ranibizumab for polypoidal choroidal vasculopathy: 6-month results. Br J Ophthalmol. 2010;94:297-301.

18. Koh A, Lee WK, Chen LJ, Tan NW, Lim TH, EVEREST Study Group. EVEREST study: efficacy and safety of verteporfin photodynamic therapy in combination with ranibizumab or alone versus ranibizumab monotherapy in patients with symptomatic macular polypoidal choroidal vasculopathy. Retina. 2012;32:1453-64.

19. Koh AH, Expert PCV Panel, Chen LJ, Chen SJ, Chen Y, Giridhar A, Iida $T$, et al. Polypoidal choroidal vasculopathy: evidencebased guidelines for clinical diagnosis and treatment. Retina. 2013;33:686-716.

20. Gomi F, Oshima Y, Mori R, Kano M, Saito M, Yamashita A, et al. Initial versus delayed photodynamic therapy in combination with ranibizumab for treatment of polypoidal choroidal vasculopathy: the Fujisan study. Retina. 2015;35:1569-76.

21. Hara C, Sawa M, Sayanagi K, Nishida K. One-year results of intravitreal aflibercept for polypoidal choroidal vasculopathy. Retina. 2016;36:37-45.

22. Maruyama-Inoue M, Sato S, Yamane S, Kadonosono K. Intravitreal injection of aflibercept in patients with polypoidal choroidal vasculopathy: a 3-year follow-up. Retina. 2017; https://doi.org/10. 1097/IAE.0000000000001818 
23. Inoue $\mathrm{M}$, Yamane $\mathrm{S}$, Taoka $\mathrm{R}$, Arakawa $\mathrm{A}$, Kadonosono $\mathrm{K}$. Aflibercept for polypoidal choroidal vasculopathy: as needed versus fixed interval dosing. Retina. 2016;36:1527-34.

24. Saito M, Kano M, Itagaki K, Oguchi Y, Sekiryu T. Switching to intravitreal aflibercept injection for polypoidal choroidal vasculopathy refractory to ranibizumab. Retina. 2014;34: 2192-201.

25. Azuma K, Obata R, Nomura Y, Tan X, Takahashi H, Yanagi Y. Angiographic findings of ranibizumab-resistant polypoidal choroidal vasculopathy after switching to a treat-and-extend regimen with intravitreal aflibercept. Retina. 2016;36:2158-65.

26. Uyama M, Matsubara T, Fukushima I, Matsunaga H, Iwashita K, Nagai Y, et al. Idiopathic polypoidal choroidal vasculopathy in Japanese patients. Arch Ophthalmol. 1999;117:1035-42.

27. Ma L, Li Z, Liu K, Rong SS, Brelen ME, Young AL, et al. Association of genetic variants with polypoidal choroidal vasculopathy: a systematic review and updated meta-analysis. Ophthalmology. 2015;122:1854-65.

28. Rouvas AA, Papakostas TD, Ntouraki A, Douvali M, Vergados I, Ladas ID. Photodynamic therapy, ranibizumab, and ranibizumab with photodynamic therapy for the treatment of polypoidal choroidal vasculopathy. Retina. 2011;31:464-74.
29. Tan CS, Ngo WK, Chen JP, Tan NW, Lim TH, EVEREST Study Group. EVEREST study report 2: imaging and grading protocol, and baseline characteristics of a randomised controlled trial of polypoidal choroidal vasculopathy. Br J Ophthalmol. 2015;99: $624-8$.

30. Gharehbagh SS, Subhi Y, Sørensen TL. Efficacy of aflibercept for polypoidal choroidal vasculopathy in Caucasians. Acta Ophthalmol. 2018;96:e94-5.

31. Heier JS, Brown DM, Chong V, Korobelnik JF, Kaiser PK, Nguyen QD, et al. Intravitreal aflibercept (VEGF trap-eye) in wet age-related macular degeneration. Ophthalmology. 2012;119: 2537-48.

32. Koh A, Lai TYY, Takahashi K, Wong TY, Chen LJ, Ruamviboonsuk $\mathrm{P}$, et al. Efficacy and safety of ranibizumab with or without verteporfin photodynamic therapy for polypoidal choroidal vasculopathy: a randomized clinical trial. JAMA Ophthalmol. 2017;135:1206-13.

33. Lee WK, Iida T, Ogura Y, Chen SJ, Wong TY, Mitchell P et al. Efficacy and safety of intravitreal aflibercept for polypoidal choroidal vasculopathy in the PLANET study: a randomized clinical trial. JAMA Ophthalmol. 2018; https://doi.org/10.1001/ja maophthalmol.2018.1804 